\title{
PESQUISA DOCUMENTAL DE PRONTUÁRIOS DE ATENDIMENTO PSICANALÍTICO DE BEBÊS: PROPOSTA DE ANÁLISE QUALITATIVA EM CINCO TEMPOS
}

\author{
Isabella Regina Gomes de Queiroz ${ }^{1,2}$ e Milena Pereira Pondé ${ }^{2}$ \\ 1 Serviço de Referência em Triagem Neonatal ²Escola Bahiana de Medicina e Saúde Pública, Brasil. \\ isabellaqueiroz@bahiana.edu.br; milenaponde@bahiana.edu.br
}

\begin{abstract}
Resumo. Introdução: O estudo investigou bebês, na condição intersexuada, acompanhados longitudinalmente, no Serviço de Referência em Triagem Neonatal (SRTN), a partir da percepção dos pais. Método: Análise qualitativa em cinco tempos, elaborado para fundamentar o estudo documental de prontuários de atendimentos psicanalíticos de bebês, cujo conteúdo constitui-se objeto de investigação do pesquisador (responsável pelos registros dos prontuários). Estabelecese cinco momentos, destacando o embricamento das dimensões teóricas, técnicas e éticas. Resultado: Verificou-se alterações na tela simbólica dos pais, graças a influências dos diferentes momentos vividos: 1. Percurso diagnóstico. 2. Período pré-cirúrgico até o momento do resultado do cariótipo. 3. Período pré-cirúrgico depois do resultado do cariótipo. 4. Período pós-cirúrgico. A temporalidade do registro mostrou-se fundamental na detecção de fases específicas para questão da intersexualidade, verificando-se como pais e mães revelaram sofrimentos e relatos específicos a cada fase enfrentada, desde o nascimento até o momento pós-cirúrgico. A compreensão de projeções, atos falhos e contradições, ao longo das narrativas, permitiu alargar a visão dos casos estudados. Conclusões: Estudar prontuários, permitiu adentrar em aspectos que uma entrevista estruturada poderia não revelar. As observações do psicólogo sobre os fatos clínicos, foram fundamentais, enquanto reveladores de conflitos, desejos expressos por atos falhos, contradições, esquecimentos.
\end{abstract}

Palavras-chave: Estudo Qualitativo; Método de Pesquisa; Registros Clínicos; Bebês; Psicanálise.

\section{DOCUMENTARY RESEARCH OF MEDICAL RECORDS OF PSYCHOANALYTIC CARE FOR BABIES: A FIVE-STEP QUALITATIVE ANALYSIS PROPOSAL}

\begin{abstract}
Introduction: The study investigated babies, in the intersexed condition, followed longitudinally, at the Neonatal Screening Reference Service (SRTN), from the parents' perception. Method: Qualitative analysis in five stages, designed to support the documentary study of medical records of psychoanalytic care for babies, whose content constitutes the object of investigation by the researcher. Five moments are established, highlighting the embracing of theoretical, technical and ethical dimensions. Result: We observed changes in the symbolic screen of the parents, due to the influences of the different moments experienced: 1. Diagnostic path. 2. Pre-surgical period until the moment of the karyotype result. 3. Pre-surgical period after the karyotype result. 4. Postsurgical period. The temporality of the registration proved to be crucial in detecting specific phases for the intersexuality issue, verifying how fathers and mothers revealed suffering and specific relation to each phase faced, from birth to the postsurgical moment. The understanding of projections, flawed acts and contradictions, throughout the narratives, allowed to broaden the view of the cases studied. Conclusions: Studying medical records, allowed to enter into aspects that a structured interview might not reveal. The psychologist's observations on clinical facts were decisives, as they reveal conflicts, desires expressed by faulty acts, contradictions, forgetfulness.
\end{abstract}

Keywords: Qualitative Study; Research Method; Clinical Records; Babies; Psychoanalysis. 


\section{INTRODUÇÃO}

A PERCEPÇÃO DE PAIS E DE PROFISSIONAIS DE SAÚDE SOBRE FILHOS/ PACIENTES COM HIPERPLASIA DA SUPRARRENAL CONGÊNITA E DESORDEM DO DESENVOLVIMENTO SEXUAL, constituiu-se em uma tese de doutorado que investigou bebês, nascidos na condição intersexuada, acompanhados longitudinalmente, em um Serviço de Referência em Triagem Neonatal (SRTN). A tese utilizou dois métodos qualitativos: um para o estudo da percepção dos profissionais de saúde e outro para o estudo da percepção dos pais sobre os seus filhos. O método denominado análise qualitativa em cinco tempos foi elaborado para subsidiar o estudo da percepção dos pais, abordado nesse artigo, a partir de análise de prontuários psicanalíticos, estruturados pelo próprio autor da pesquisa. A clínica psicanalítica de bebês, é permeada pelo seu cuidador primário, geralmente, seus pais. A maneira como o bebê está inscrito na tela simbólica de seus pais, as fantasias que o envolve, a maneira como carregado, cuidado, consistem em elementos fundamentais para essa clínica. Estudar os documentos gerados a partir de atendimentos psicanalíticos consiste em um desafio para o pesquisador, posto que os dados são sigilosos e apenas o indivíduo que gerou o documento poderá ter acesso a eles, a partir do consentimento dos sujeitos/pacientes ou responsáveis (no caso de menores de idade e de vulneráveis). Ademais, o conteúdo a ser estudado não se resume às narrativas do analisando, senão, àquilo que se denomina de fato clínico. Nesse sentido, os registros decorrentes dos acontecimentos no setting clínico, estão perpassadas pelo analista: os recortes por ele feito (relacionados ao tema que se quer investigar), os elementos transferenciais, pontuações acerca das contradições, atos falhos e outros elementos presentes nesse contexto (Dallazen et al,I 2012). Dada a escassez de modelos metodológicos para esse tipo de estudo, objetiva-se, com essa proposta, estabelecer fundamentos para o estudo documental de prontuários gerados na clínica psicanalítica de bebês e crianças pequenas, atendidos longitudinalmente, cujos registros foram gerados pelo profissional responsável pelo atendimento e que, em um segundo momento, constitui-se como pesquisador a partir do material por ele elaborado.

\section{METODOLOGIA}

São estabelecidos como pressupostos da fundamentação metodológica, para o modelo de pesquisa aqui proposto, a demarcação de cinco momentos distintos. Esses momentos são 
reveladores do quanto as dimensões teóricas, técnicas e éticas são justapostas nesse tipo de investigação.

\subsection{O primeiro momento, ou a pré-pesquisa}

Corresponde ao momento dos atendimentos psicológicos dos pacientes. Nessa etapa, o documento é gerado visando, exclusivamente, o registro clínico. Ressalte-se que os registros são estruturados a partir de atendimentos clínicos, no qual o profissional adota uma posição da escuta psicanalítica. Desse modo, compreende-se que o discurso do paciente é priorizado em entrevistas abertas, com marcações necessárias ao deslizamento do discurso. São acrescidos, ainda, os registros do psicanalista referente à dimensão psíquica, observada durante a sessão, nos quais constam o parecer do profissional.

Com relação a esse momento, ressalta-se duas especificidades. A primeira referente à abordagem teórica adotada pelo autor dos registros clínicos (que posteriormente se torna pesquisador dos mesmos prontuários), que é a psicanálise. Essa primeira especificidade fica bem estabelecida através das considerações de Lacan (1957/1998; 1966/1998), que sugere uma torção para o cogito cartesiano que repousa na ideia do "penso, logo existo". O autor apresenta uma demarcação clara entre ser e pensar ao propor uma inversão para esse cogito: "lá onde penso, não sou" apontando para a importância de se considerar a dimensão inconsciente - mantida fora da ciência cartesiana. A segunda especificidade diz respeito ao local onde se desenvolve a pesquisa. Esse aspecto é de fundamental importância, já que o estabelecimento de protocolos delimita a dinâmica da clínica, desde o fazer do profissional, responsável pelos atendimentos, à dimensão transferencial.

$\mathrm{Na}$ clínica psicanalítica, as descrições nos prontuários são realizadas a posteriori ao momento da consulta e, assim, a experiência clínica perpassa o analista e desdobra-se sobre os registros: os recortes por ele feito, as interpretações, a leitura da cena apresentada no setting analítico, as pontuações dos tropeços de linguagem constituindo, dessa maneira, o que se denomina de fatos clínicos - fatos tratados durante a comunicação constituída no campo psicanalítico , que é o "campo transferencial, onde a análise se dá, marcado pela operação fundamental do analista: a interpretação. (Herrmann \& Herrmann, 2012). Do lado do analisante, abarca-se toda a comunicação esta advinda de distintas situações: experiência vivida/sentida/imaginada, sonhos, atos. Do lado do analista, abarca-se a sua própria experiência, além da técnica e do arcabouço teórico (Vollmer Filho, 1994). 
Para a proposta em questão, é de fundamental importância analisar as especificidades de uma clínica de bebês (devir de sujeito). Compreende-se que as questões vivenciadas nos sistemas mãe-bebê/mãe-pai-bebê, não estão dadas pelo fato vivido, apenas, na dimensão real do corpo do bebê. Ocorre, também, nas dimensões imaginária e simbólica, na medida em que os sonhos e as idealizações em torno do bebê, tanto quanto a maneira como este é falado, estão entrelaçadas nas antecipações do Outro (encarnado, geralmente, na mãe) e nas distintas atribuições de sentido, como diz Aulagnier (1990; 2000) que vão sendo tecidas, enquanto os cuidados ao bebê vão sendo prestados. Trata-se de favorecer um olhar para além da dimensão biológica, defendendo a possibilidade de se construir um saber sobre esse filho, desde a sua pré-história, seguindo com os elementos psíquicos constituídos a partir de um sistema estabelecido na díade ou na tríade, em suas distintas instâncias.

Os registros produzidos nessa clínica, constituem-se regularmente de fatos clínicos e estes estão sempre vinculados ao método de apreensão destes e aos seus fundamentos teóricos (Quinodoz, 1994). Nesse tempo inicial, que se constitui a condição sine qua non dessa modalidade de pesquisa, em não há nenhuma pergunta de investigação e nenhum objeto de pesquisa estipulados, apenas os registros de prontuários são produzidos.

\subsection{0 segundo momento}

Possibilita uma apropriação das construções realizadas ao longo dos atendimentos, ora ressignificadas a partir da delimitação do que se quer investigar. É, portanto, um momento marcado pela rememoração de conteúdos psíquicos presentes no investigador do que fora construído a partir das experiências clínicas com os seus pacientes, agora, transformados em pretensos casos a serem estudados.

Está em jogo aqui a questão da temporalidade, uma vez que é apenas em "um só depois" que esses novos sentidos se instituem - como em um "APRÉS COUP” (Laplanche, 2016).

A teoria é posta em uma condição de suspensão para que seja possível insurgir o novo condição necessária, também, ao trabalho clínico no que concerne a relação do psicanalista como seu paciente (e, portanto, presente, também, no primeiro momento). É nesse instante que o profissional responsável pelos acompanhamentos clínicos assume a posição de pesquisador. As experiências vividas nessa etapa preparam para o momento seguinte. 


\subsection{0 terceiro momento}

Consiste no tempo em que ocorre o estabelecimento da pergunta de investigação e do objeto de pesquisa. A experiência do momento anterior é de fundamental importância para esse terceiro momento, pois é ela quem aparelha o investigador a inúmeras elaborações que irão fundamentar o seu interesse de estudo. Assim, deixar em suspenso a teoria, abre possibilidades de novos sentidos que poderão emergir na elaboração da pergunta de pesquisa e do objeto a que esta se refere.

\subsection{0 quarto momento}

Marca a pesquisa propriamente dita. A leitura dos documentos, a partir da técnica da atenção flutuante, possibilita a organização de sentidos relacionando os conteúdos acessados nos prontuários à pergunta de investigação, produzindo um novo desdobramento para a dimensão semântica, produzida na etapa antecedente. Esse quarto momento é destinado a examinar as vivências psíquicas registradas no material clínico em questão, possibilitando a produção de conhecimento, por parte do pesquisador, em uma outra instância, na qual a relação de transferência se apresenta fora do setting clínico. Esse reposicionamento favorece, um "sobre-olhar" para os conteúdos registrados, fazendo-se ver as narrativas dos pais referentes ao paciente e, também, os fatos clínicos (que como dito anteriormente compõe o registro em questão).

Nesse instante, a observação participante torna-se a eleita para se constituir o tipo de observação coerente com os propósitos desse modelo de pesquisa. Nessa direção, os registros devem ser tomados como uma aproximação à ideia de um diário de campo justificada a partir da noção da observação participante; mas tomados, apenas, como uma aproximação, já que esses prontuários são registros elaborados em um tempo anterior ao estabelecimento da pesquisa.

Chamaremos, então, de "Diário de Campo Livre", por se tratar de anotações que não tiveram como objetivo a pesquisa, pois foram registradas livres de uma ideia relacionada a um objeto de pesquisa específico.

Esse momento encontra-se, de certa maneira, entrelaçado ao anterior, já que nele deve-se manter, ainda, todo arcabouço teórico do pesquisador em suspensão, considerando-se, também, a ideia de “Après Coup" (Freud, 1996; Laplanche, 2016). 


\subsection{0 quinto momento}

Instante da investigação propriamente dita, é destinado à Categorização, Análise e Interpretação do material contido na entrevista.

Sendo a clínica aqui tratada, uma clínica que envolve bebês e crianças pequenas a dimensão ética envolve algumas especificidades que devem atender a determinados requisitos éticos. $\mathrm{O}$ acesso aos conteúdos dos prontuários, para fins de pesquisa, deve ocorrer mediante autorização prévia dos responsáveis pela criança e, em casos de pacientes com mais de quatro anos, o assentimento para esse acesso constitui-se em um dos critérios ético fundamental.

Ao retomar as anotações de prontuário, o documento produzido pelo próprio investigador é relido, mas, agora, à luz da pergunta de investigação atual. Para tanto, vale-se da técnica da atenção flutuante, (como no momento anterior) na busca de categorias emergentes dos conteúdos dos documentos, iniciada no momento anterior e seguindo-se no momento atual. Caracterizado pelo início da investigação propriamente dita, esse momento é marcado por três etapas que se entrelaçam: 5.1) a organização de Categorias, a partir das narrativas estudadas, 5.2) a Análise do material encontrado e 5.3) a Interpretação, que será feita à luz da psicanálise.

5.1) A organização de Categorias: Coerentemente com os fundamentos da análise de dados qualitativos em dois níveis (Pondé, Mendonça \& Caroso, 2009), após a leitura flutuante dos documentos, procede-se uma segunda leitura, essa agora, sistemática, buscando relacionar fragmentos do documento/texto com ideias-chave, que chamaremos de categorias. Os fragmentos dos documentos, então, são marcados e ligados a Categorias, que vão sendo criadas durante a Segunda Leitura Sistemática. Procede-se, então, uma Terceira Leitura Sistemática dos documentos, para permitir que todos os documentos possam ser categorizados conforme todas as Categorias geradas ao longo da Segunda Leitura Sistemática dos documentos. Em seguida são criados arquivos para cada categoria: o arquivo tem o nome da Categoria e contém todos os fragmentos marcados em cada documento referente àquela Categoria, sendo que cada fragmento deve ser identificado, exemplo: Fragmento do Documento 1, Fragmento do Documento 2 e assim sucessivamente. O momento seguinte será reler os fragmentos de cada Arquivo de Categoria, criando então Subcategorias. Subsequentemente, procede-se a análise e posterior interpretação dos dados. 
5.2) A etapa de Análise das categorias consiste em identificar padrões ou tendências nas Subcategorias. Requer que o pesquisador tenha profundo conhecimento dos documentos da pesquisa, porque a identificação de padrões deve ser feita contemplando os "fatos clínicos", acima mencionados, que podem estar presentes nas anotações do clínico/pesquisador (autor dos documentos) e podem surgir "aprés-coup" na lembrança do pesquisador (o próprio autor do documento no Primeiro Momento, ou pré-pesquisa.

O processo de categorização consiste em um exercício criterioso, porque os textos dos prontuários não respondem a perguntas específicas e dessa maneira, a cadeia das narrativas passeia por temas que se interligam (ou não) segundo a subjetividade de quem narra e a própria dinâmica das sessões clínicas. Podem, então, conter dados explícitos e implícitos e só o trabalho de análise desse material possibilitará a adequação da organização dos dados. Além disso, não há uma padronização no curso das 'respostas' à pergunta de investigação. Compreende-se que a ênfase dada a determinados pontos durante as narrativas, em detrimento de outros, também consiste em dado de análise. Esse aspecto dos registros, inclusive a falta de padronização no curso das respostas, aponta para a possibilidade de lacunas de elementos que compõem a pesquisa. Nesse momento, valerse da observação participante auxilia na estruturação melhor dos dados pesquisados, incluindo esses registros no item percepção do psicólogo (o que deve constituir em um item específico no momento da análise). Nessa sub-etapa da pesquisa, a discussão com um terceiro, posto fora da cena clínica, favorece a manutenção de um sobreolhar, presente desde o momento anterior e fundamental para a ampliação semântica no momento da categorização.5.3)

\subsection{A Interpretação à luz da psicanálise}

Caracteriza-se pela Interpretação do material encontrado e corrobora com o próprio percurso da história da psicanálise, no sentido de ela ter se estruturado na constante articulação dos achados clínicos de Freud e suas frequentes revisitações à dimensão teórica. O Processo de interpretação vale-se do espaço concedido pelo próprio autor da psicanálise, para a articulação de saberes. A história da psicanálise se fez em movimento contínuo segundo o qual o seu autor compreende sua teoria/prática como um saber aberto, constantemente revisitado a partir dos estudos de casos atendidos e por ele analisados, ao tempo em que indica a importância de um saber científico que considere essas relações nas dimensões: tópica, dinâmica e econômica do psiquismo, ao tempo em que reforça a 
compreensão do saber psicanalítico, desde sua técnica, como um saber aberto. Diz o autor: "Penso estar sendo prudente, contudo, em chamar estas regras de 'recomendações' e não reivindicar qualquer aceitação incondicional para elas. (...) Freud, 1913, p. 164). Essa brecha, forjada pelo autor, funciona como uma convocatória para um movimento no fazer clínico, apontando para possibilidades posteriores de novas articulações teórico-práticas.

A pesquisa A PERCEPÇÃO DE PAIS E DE PROFISSIONAIS DE SAÚDE SOBRE FILHOS/ PACIENTES COM HIPERPLASIA DA SUPRARRENAL CONGÊNITA E DESORDEM DO DESENVOLVIMENTO SEXUAL foi aprovada pelo Comitê de Ética e Pesquisa da Fundação Bahiana para o Desenvolvimento das Ciências, 11/08/2016 e número 1672790 para o estudo de pacientes; e em 14/05/2017 e número 1963747 para o estudo dos profissionais de saúde - visando assegurar a defesa e a proteção dos sujeitos envolvidos, que assinaram o Termo de Consentimento Livre Esclarecido, seguindo a resolução do CONEP 2012.

\section{RESULTADOS}

O cumprimento das cinco etapas propostas pelo método aqui apresentado mostrou-se fundamental para a análise da dimensão psíquica que envolve a questão do nascimento de um filho na condição intersexual até o momento pós genitoplastia, favorecendo a apropriação de processos conscientes e inconscientes dos pais, que vivenciaram situação traumática nesse percurso. Foi possível avaliar a alteração da tela simbólica a partir de influências dos diferentes momentos vividos, destacados nos prontuários e a análise dos desdobramentos dessa alteração sobre o filho, retratados nas categorias construídas a partir do estudo dos prontuários.

1. Percurso diagnóstico:

Marcado por sentimentos ambivalentes: de um lado, amor, o medo da morte do bebê, de outro, sentimentos de: rejeição, dor, vergonha em decorrência da ambiguidade dos genitais. Verificou-se sonhos desfeitos e resistências. O sofrimento psíquico e o adoecimento (depressão e ansiedade) fizeram-se presentes. Foram narradas distintas formas da equipe abordar a questão da HSRC: maus tratos, curiosidades, cuidados, erros;

2. Período pré-cirúrgico até o momento do resultado do cariótipo:

Momento de confusão em relação à designação sexual da criança, levando os pais a tratarem e nomearem os bebês de maneira concebida por eles como ambígua. Percepção de anormalidade no filho e estabelecimento de segredo mediante a condição do bebê. O discurso médico se sobrepôs à tela simbólica dos pais, em certo nível, levando a alterações das antecipações acerca do bebê, tão caras à constituição psíquica; 


\section{Período pré-cirúrgico depois do resultado do cariótipo:}

Caracterizado pela a definição do sexo (46XX) possibilitou o registro civil da criança (em suspenso em alguns casos) e /ou de solicitação judicial para a mudança do registro anterior em casos de mudança do sexo. Verificou-se mobilização psíquica intensa nos pais, naqueles casos em que o sexo do bebê foi alterado, desdobrando-se na maneira de olhar e cuidar da criança;

\section{Período pós-cirúrgico:}

Momento não vivenciado por todos os investigados; cinco aderiram à genitoplastia. Dentre esses, observou-se certo apaziguamento inicial, sucedendo-se questões relativas à sexualidade futura da filha, quanto: à identidade de gênero, à orientação sexual e, mesmo, ao gênero (duvidando do saber médico). Verificou-se: atos falhos, conferindo questões relativas ao desejo, com trocas pronominais referente ao gênero da criança, além de projeção da mãe em relação à filha, quanto aos seus próprios conteúdos psíquicos:

“(...) o povo falava: 'pelo jeito de M4, vai ser menino'. Porque falava que eu tenho jeito de homem (...) eu acho que ela é meu jeito.

A intersexualidade que se pretendia apagar, com a cirurgia no real do corpo, tendeu a retornar, minimamente, como questão (acentuadas pela cicatriz e pelo uso de medicamentos indicados para HSRC).

\section{DISCUSSÃO}

A temporalidade do registro se mostrou fundamental para a detecção de fases específicas para a questão estudada: é o fluxo dos atendimentos que revela que o tempo precoce para a cirurgia não, necessariamente, garante o apaziguamento em relação à ambiguidade genital - o tempo de elaboração das questões relaciona-se com a ideia de tempo lógico. A identificação de atos falhos e contradições nos discursos, ao longo do processo, puderam apontar para questões psíquicas envolvidas na designação sexual das crianças, fundamentais para os resultados da investigação em questão. As observações do psicólogo sobre os fatos clínicos observados, apresentaram a importância de se pensar nesse construto, enquanto revelador de conflitos, desejos expressos por atos falhos, contradições, esquecimentos. Essa ideia fica contemplada pela análise em dois níveis utilizada no método, proposta por Pondé (2009). Reforça-se o conceito de diário de livre de campo, emergido das observações dos registros psicológicos, importantes para a análise de dados como esses.

\section{COMENTÁRIOS FINAIS}

Os achados reforçam a ideia de que tomar o estudo documental em cinco tempos como modelo de pesquisa documental possibilitou que presente estudo pudesse responder 
adequadamente à questão estabelecida para a pesquisa "Percepção de pais e de profissionais de saúde sobre filhos/ pacientes com hiperplasia da suprarrenal congênita e desordem do desenvolvimento sexual." Os estudos dos prontuários dos pacientes, analisados longitudinalmente, permitiram uma incursão em aspectos que uma entrevista estruturada para pesquisa científica poderia não revelar: a o percurso dos relatos em cada etapa vivenciada. As observações do psicólogo sobre os fatos clínicos observados, apresentaram a importância de se pensar nesse construto, enquanto revelador de conflitos, desejos expressos por atos falhos, contradições, esquecimentos. Essa ideia fica contemplada pela análise em dois níveis utilizada no método, proposta por Pondé (2009). Reforça-se o conceito de diário de livre de campo, emergido das observações dos registros psicológicos, importantes para a análise de dados como esses.

\section{REFERÊNCIAS}

Aulagnier, P. (2000) Lo potencial, lo posible, lo imposible: categorías y coordenadas del campo clínico. Psicoanálisis AP de BA, 22, 65-87.

Dallazen, L.; Giacobone, R.V.; Macedo, M. M. K. \& Kupermann (2012), D. Sobre a ética em pesquisa na psicanálise. Psico, Porto Alegre, PUCRS, 43(1), pp. 47-54.

Freud, S. (1980). Sobre o Início do Tratamento. (Novas recomendações sobre a técnica da psicanálise I). Em Obras Completas. v. 12. Rio de Janeiro: Imago (Trabalho original publicado em 1913).

Freud, S. (1996). Recordar, repetir e elaborar. In S. Freud, Edição standard brasileira das obras psicológicas completas de Sigmund Freud. (Vol.12). Rio de Janeiro: Imago. (Trabalho original publicado em 1914)

Herrmann, F. \& Herrmann, L. (2012). Notas sobre campo e campo psicanalítico. Ide (São Paulo), São Paulo, 35 (54), $139-147$.

Lacan, J. (1998). A instância da letra no inconsciente ou a razão desde Freud. Em: Escritos (496-533). Rio de Janeiro: Jorge Zahar. (Texto original publicado em 1957a).

Laplanche, Jean; Pontalis, Jean-Bertrand. Vocabulário da psicanálise. 4. ed. São Paulo: Martins Fontes, 2016. (Trabalho original publicado em 1967)

Pondé, M. P., Mendonça, M. S. S., \& Caroso, C. (2009). [Methodological proposal for a dual a level analysis of qualitative data]. História, Ciências, Saúde-Manguinhos, 16(1), 129-143.

Quinodoz, J. M. (1994). Fatos clínicos ou fatos clínicos psicanalíticos? Revista Brasileira de 28(4):613-34.

Psicanálise,

Vollmer Filho, G. (1994). A conceituação do fato clínico psicanalítico. Revista Brasileira de Psicanálise, 28(4), 673-85. 